\title{
Autoavaliação ruim do estado de saúde: prevalência e fatores associados em mulheres privadas de liberdade ${ }^{a}$
}

Poor self-rated health: prevalence and associated factors on women deprived of liberty Autoevaluación negativa de la salud: prevalencia y factores asociados en mujeres privadas de libertad

Lidiane Castro Duarte de Aquino ${ }^{1}$ (D) Bruna Gomes de Souza ${ }^{2}$ (1) Cosme Rezende Laurindo 3 (B) Isabel Cristina Gonçalves Leite 3 (1) Danielle Teles da $\mathrm{Cruz}^{3}$ (D)

1. Universidade Federal de Juiz de Fora. Juiz de Fora, MG, Brasil.

2. Universidade Federal de Juiz de Fora, Faculdade de Medicina. Juiz de Fora, MG, Brasil.

3. Universidade Federal de Juiz de Fora, Programa de Pós-Graduação em Saúde Coletiva. Juiz de Fora, MG, Brasil.

Autor correspondente:

Danielle Teles da Cruz.

E-mail: danitcruz@yahoo.com.br

Recebido em 15/07/2021.

Aprovado em 18/10/2021.

DOl:https://doi.org/10.1590/2177-9465-EAN-2021-0275

\section{Resumo}

Objetivo: Verificar a prevalência da autoavaliação ruim do estado de saúde em mulheres encarceradas e analisar os fatores associados. Método: Trata-se de estudo transversal, realizado entre os anos de 2019 e 2020, por meio de censo, com participação de 99 mulheres. A análise dos fatores associados ao desfecho foi conduzida a partir de um modelo teórico de determinação com três blocos hierarquizados de variáveis. As variáveis foram ajustadas entre si dentro de cada bloco. Aquelas com nível de significância $\leq 0,20$ foram incluídas no modelo de regressão de Poisson e ajustadas ao nível superior ao seu considerando o nível de 5\% de significância. Resultados: A prevalência da autoavaliação ruim da saúde foi de 31,3\% (IC95\% = 22,8\%-40,9\%). Morbidade referida, presença de sintomas de ansiedade e a pior perspectiva em relação às condições de saúde pós-encarceramento foram as variáveis associadas com o desfecho. Considerações finais e implicações para a prática: Os fatores associados à ocorrência do evento investigado poderão direcionar medidas que visem à redução dos impactos à saúde durante o período de encarceramento.

Palavras-chave: Autoavaliação; Estudos Transversais; Mulheres; Prisões; Saúde.

\section{Abstract}

Objective: To verify the prevalence of poor self-rated health status among incarcerated women and to analyze the associated factors. Method: This is a cross-sectional study, carried out between 2019 and 2020, by means of a census, with the participation 99 women incarcerated. The analysis of factors associated with the outcome was conducted based on a theoretical model of determination with three hierarchical blocks of variables. Variables were adjusted to each other within each block. Those with significance level $\leq 0.20$ were included in the Poisson regression model and adjusted to a level higher than theirs, considering a $5 \%$ level of significance. Results: The prevalence of poor self-rated health was $31.3 \%\left(\mathrm{IC}_{95 \%}=22.8 \%-40.9 \%\right)$. Reported morbidity, presence of anxiety symptoms and the worst perspective regarding post-incarceration health conditions were the variables associated with the outcome. Conclusion and implications for practice: The factors associated to the occurrence of the investigated event may direct measures aimed to reduce health impacts during the incarceration period.

Keywords: Cross-Sectional Studies; Health; Prisons; Self-Assessment; Woman

\section{Resumen}

Objetivo: Verificar la prevalencia de autoevaluación negativa de la salud en mujeres encarceladas y analizar los factores asociados. Método: Se trata de un estudio transversal, realizado entre los años 2019 y 2020, mediante censo, con la participación de 99 mujeres encarceladas. El análisis de factores asociados al resultado se realizó con base en un modelo teórico de determinación con tres bloques jerárquicos de variables. Las variables se ajustaron entre sí dentro de cada bloque. Aquellos con nivel de significancia $\leq 0,20$ se incluyeron en el modelo de regresión de Poisson y se ajustaron a un nivel superior al de ellos, considerando un nivel de significancia del 5\%. Resultados: La prevalencia de autoevaluación negativa de la salud fue 31,3\% (IC 95\% = 22,8\% $-40,9 \%$ ). La morbilidad autoinformada, la presencia de síntomas de ansiedad y la peor perspectiva con respecto a las condiciones de salud después del encarcelamiento fueron las variables asociadas con el resultado. Conclusión e implicaciones para la práctica: Los factores asociados a la ocurrencia del evento investigado pueden conducir a medidas destinadas a reducir los impactos en la salud durante el período de encarcelamiento.

Palabras clave: Autoevaluación; Estudios Transversales; Mujeres; Prisiones; Salud. 


\section{INTRODUÇÃO}

Embora as mulheres representem aproximadamente 5\% da população carcerária brasileira, a taxa de encarceramento feminino cresceu de forma significativa ao longo dos anos. De acordo com os dados do Departamento Penitenciário Nacional, entre 2000 e 2016, a população prisional feminina aumentou em $656 \%$, enquanto que, no mesmo período, o encarceramento masculino cresceu $293 \%{ }^{1}$.

A maioria das mulheres encarceradas apresenta situação prévia de vulnerabilidade social, sendo jovens, negras, solteiras, com filhos, baixo nível de escolaridade e renda familiar precária1. Além disso, diversos estudos ressaltaram a influência negativa do encarceramento na saúde de mulheres privadas de liberdade, seja no aspecto do bem-estar físico, mental ou social ${ }^{2-4}$. A partir da perspectiva de compreensão da saúde como uma complexa produção social, admite-se que essas condições podem contribuir para uma trama de vínculos e associações que predispõe a autoavaliação ruim do estado de saúde.

A autoavaliação da saúde vem sendo amplamente utilizada em estudos transversais ${ }^{5-14}$ tanto pela sua facilidade de captação quanto por sua validade e relevância enquanto medida de saúde. O indicador está associado a uma concepção individual e subjetiva acerca da saúde, sob influência de aspectos biológicos, psicológicos e sociais ${ }^{15}$, aproximando-se, portanto, de uma concepção mais ampliada da saúde. Tem sido utilizado como um marcador de desigualdades entre subgrupos populacionais, para descrever o estado de saúde das populações, estabelecer diferenças de morbidade em subgrupos populacionais, comparar necessidade por serviços de saúde e recursos, além de ser um bom preditor de morbi-mortalidade $e^{7,8,15}$.

$\mathrm{O}$ indicador tem sido utilizado em escala internacional e nacional, sendo que, internacionalmente, há evidências que apontam para a associação entre a pior percepção do estado de saúde e idade, nível de escolaridade e de renda ${ }^{5,16-19}$. Já a nível nacional, estudos confirmam forte associação entre condições socioeconômicas desfavoráveis e pior percepção do estado de saúde ${ }^{7,9,10}$. Além disso, diferenças entre gêneros, faixas etárias, estilo de vida e condições ambientais também são variáveis que influenciam a autoavaliação da saúde ${ }^{11-14}$. Essas evidências demonstram a importância e a potência do uso do indicador nos mais variados contextos.

Embora a autoavaliação do estado de saúde já tenha sido explorada em diversos grupos, seu uso na população privada de liberdade ainda é incipiente. No entanto, há evidência que aponta para o papel vital que as instituições de privação de liberdade podem desempenhar sobre a forma como os indivíduos ali presentes avaliam a própria saúde ${ }^{20,21}$. Sendo assim, levando em consideração a robustez do indicador, bem como as especificidades que o aprisionamento impõe, acreditamos ser esse um elemento de análise importante para a compreensão do processo saúdeadoecimento das mulheres privadas de liberdade.

Dessa forma, o objetivo do presente estudo foi estimar a prevalência e os fatores associados à autoavaliação ruim do estado de saúde em mulheres encarceradas em um estabelecimento penal de Juiz de Fora, Minas Gerais.

\section{MÉTODO}

Trata-se de um estudo epidemiológico de delineamento transversal, do tipo censo, entre setembro de 2019 e fevereiro de 2020, com as mulheres reclusas no Anexo Feminino Eliane Betti em Juiz de Fora, Minas Gerais. Atualmente, o local é a única referência para o acautelamento feminino entre os mais de 80 municípios que compõem a 4ª Região Integrada de Segurança Pública (RISP) de Minas Gerais.

Foram incluídas no estudo todas as mulheres privadas de liberdade, com idade mínima de 18 anos, independente do regime penal que se encontravam e com tempo de reclusão de no mínimo 30 dias ou que viessem a completar 30 dias no decorrer da pesquisa. Os critérios de exclusão estabelecidos foram: acauteladas que não se comunicassem através da língua padrão portuguesa; impossibilidade de compreensão e/ou resposta ao questionário; presença de distúrbios severos que afetassem a comunicação; e aquelas que, de acordo com a direção da unidade, pudessem oferecer risco à segurança da pesquisadora.

No primeiro dia de entrevistas, foi disponibilizada pela unidade prisional uma listagem na qual constavam 134 mulheres matriculadas na unidade. Novas listagens foram emitidas mensalmente, a fim de acompanhar o dinamismo de admissões e desligamentos, e, consequentemente, a possibilidade de inclusão de novas participantes ao estudo e monitoramento de possíveis perdas. Ao fim do período de coleta, a população acessível foi contabilizada em 150 mulheres.

A rotina da coleta de dados foi planejada de forma a assegurar que todas essas mulheres participassem do estudo. Quando não foi possível a abordagem no primeiro agendamento, foram realizadas três novas tentativas em dias e horários distintos. As perdas perfizeram um total de 51, sendo 21 por motivo de alvará de soltura, 4, por transferências para outras unidades prisionais e 26 não puderam participar em decorrência da interrupção da coleta devido à pandemia de COVID-19. Não houve recusas. Assim sendo, a amostra final foi composta por 99 mulheres.

A coleta de dados foi realizada uma vez por semana, através de entrevista face a face, nas salas de atendimento da unidade prisional, no Núcleo de Assistência à Saúde (NAS) e nas dependências de uma fábrica de trabalho. As policiais penais garantiram o procedimento de trânsito das detentas e a supervisão do lado externo das salas.

O questionário utilizado foi composto por questões semiestruturadas, elaboradas a partir do instrumento utilizado na pesquisa Estudo das condições de saúde e qualidade de vida dos presos e das condições ambientais das unidades prisionais do Estado do Rio de Janeiro ${ }^{22}$ e por escalas padronizadas e de ampla utilização em pesquisas científicas, como o Patient Health Questionnaire-4 (PHQ-4), para rastreio dos sintomas de ansiedade e depressão, e a Escala MacArthur de Status Social Subjetivo, para avaliação do status social subjetivo. $O$ 
instrumento de coleta foi padronizado e pré-testado em estudo piloto realizado no Presídio de Eugenópolis, com cerca de $10 \%$ da amostra prevista.

O PHQ-4 consiste em um instrumento para avaliação do sofrimento psíquico, contendo quatro itens com pontuação a partir de uma escala de Likert de zero (nenhuma vez) a três (quase todos os dias). Maior pontuação total reflete maior sintomatologia. Os dois primeiros itens compõem a subescala de ansiedade, e os outros, a subescala de depressão. O somatório dos itens de cada subescala varia de zero a seis, sendo escore $\geq$ três considerado positivo ${ }^{23,24}$. Quanto à Escala de MacArthur, ela é apresentada visualmente por uma escada com 10 degraus e foi desenvolvida com o objetivo de capturar a percepção de senso comum de status social, tendo como referência indicadores socioeconômicos. Nela, o entrevistado indica o degrau que considera representar o seu lugar na sociedade. Quanto menor a posição referida, menor o status social subjetivo. Para análise, as categorias foram agrupadas em pares: muito ruim (degraus 1 e 2), ruim (3 e 4), regular (5 e 6), bom (7 e 8) e muito bom (9 e 10). Posteriormente, foram categorizadas em pior status social subjetivo (considerando as categorias muito ruim, ruim e regular) e melhor status social subjetivo, agrupando as categorias muito bom e bom ${ }^{25}$.

A variável dependente foi a autoavaliação da saúde, obtida por meio da pergunta: Em geral, você diria que sua saúde é? As opções de resposta apresentadas foram muito boa, boa, regular, ruim e muito ruim. Para a análise do desfecho autoavaliação ruim da saúde, a variável foi dicotomizada em ruim (regular, ruim e muito ruim) e boa (boa e muito boa).

Os dados foram organizados e processados em um banco de dados criado no software Statistical Package for Social Sciences (SPSS), versão 15.0, e submetidos à análise descritiva para a obtenção de frequências absoluta e relativa, assim como da prevalência do desfecho investigado. A associação entre as variáveis independentes e a variável dependente na análise bivariada foi analisada a partir do Teste Qui-Quadrado. Já na análise multivariada, a associação entre as variáveis foi analisada a partir da regressão de Poisson, controlando as variáveis independentes por possíveis fatores de confusão (RP ajustada). O nível de significância do estudo foi de $5 \%(p \leq 0,05)$.

Para a análise dos fatores associados à autoavaliação ruim do estado de saúde, foi construído um modelo teórico de determinação ${ }^{26}$, com três blocos hierarquizados de variáveis (Figura 1), as quais foram ajustadas entre si dentro de cada bloco, em um primeiro momento. Aquelas variáveis que alcançaram um nível de significância $\leq 0,20$ foram incluídas no modelo de regressão de Poisson e ajustadas ao nível superior ao seu.

As variáveis independentes foram agrupadas nos três blocos: Bloco 1, com características demográficas e socioeconômicas; Bloco 2, composto por questões relacionadas ao encarceramento (dividido em quatro subníveis: 2.1 características penais; 2.2 assistência e recebimento de visitas; 2.3 discriminação e violência; 2.4 expectativas pós-encarceramento); e o Bloco 3 , com variáveis relacionadas à saúde das mulheres, dividido em
3 subníveis: 3.1 variáveis de saúde referidas; 3.2 variáveis de saúde autopercebidas; e 3.3 serviços de saúde no cárcere.

Este estudo está inserido em um projeto mais amplo, denominado Condições de Vida e de Saúde de Mulheres Privadas de Liberdade em Juiz de Fora, Minas Gerais. Foram respeitadas as recomendações presentes nas Diretrizes e Normas Regulamentadoras de Pesquisa Envolvendo Seres Humanos, constadas na Resolução 466 do Conselho Nacional de Saúde. O Comitê de Ética e Pesquisa da Universidade Federal de Juiz de Fora aprovou o estudo (Parecer no 3.294.253). O Termo de Consentimento Livre e Esclarecido foi lido e assinado por todas as participantes.

\section{RESULTADOS}

Participaram do estudo 99 mulheres. Aproximadamente $76 \%$ encontravam-se na faixa etária de 20 a 39 anos, sendo a média de idade de 33,21 anos (DP $\pm 9,26$ ). As que relataram serem casadas ou terem um (a) companheiro (a) totalizaram 53,5\%. A maioria declarou cor de pele preta ou parda $(74,7 \%)$, renda mensal menor ou igual a um salário mínimo no período anterior ao encarceramento $(66,7 \%)$ e com 5 a 8 anos de escolaridade $(57,6 \%)$. Quanto ao status social subjetivo, $81,9 \%$ considerou estar nas posições mais inferiores da escala utilizada (entre o $1^{\circ}$ e 5o degrau).

O tráfico de drogas foi o responsável por $52,5 \%$ das prisões. Aproximadamente $30 \%$ das entrevistadas estavam detidas provisoriamente. Dentre as sentenciadas, a maioria relatou pena a cumprir menor ou igual a sete anos $(51,4 \%)$. O recebimento de visitas foi informado por $60,6 \%$, e nenhuma participante relatou recebimento de visita íntima. Apenas 12,1\% estudavam e 40,4\% estavam inseridas em alguma atividade laboral. Todas as participantes relataram ter sofrido algum tipo de discriminação na unidade prisional. Em relação aos riscos a que estão expostas, 63,6\% reportaram o risco de sofrer violência psicológica, e 49,5\%, de sofrer agressão física. Quanto às ações em que foram vítimas, 46,5\% declararam já ter sofrido agressão verbal (Tabela 1).

A prevalência da autoavaliação ruim do estado de saúde foi de $31,3 \%$ (IC95\% = 22,8\%-40,9\%). Dentre as categorias utilizadas, $10,1 \%$ autoavaliaram a saúde como muito boa, $58,6 \%$, como boa, $23,2 \%$, como regular, $5,1 \%$, como ruim e $3,0 \%$, como muito ruim. A presença de morbidades foi referida por $52,5 \%$ das mulheres; $70,7 \%$ declararam uso contínuo de medicações; $28 \%$ informaram a ocorrência de pelo menos um aborto na vida; e 72,7\% são fumantes. Sintomas de ansiedade e depressão foram observados em, respectivamente, $75,8 \%$ e $65,7 \%$ das participantes (Tabela 2 ).

Na análise bivariada, a autoavaliação ruim da saúde esteve associada ao recebimento de tratamento preconceituoso por outras detentas, relato do risco de explosão da unidade prisional e ocorrência de queda. Foi frequente também entre aquelas que possuem pior perspectiva em relação às condições de saúde após o encarceramento ( $p<0,001)$, entre as que referiram morbidades $(p<0,002)$ e entre aquelas que apresentam sintomas 


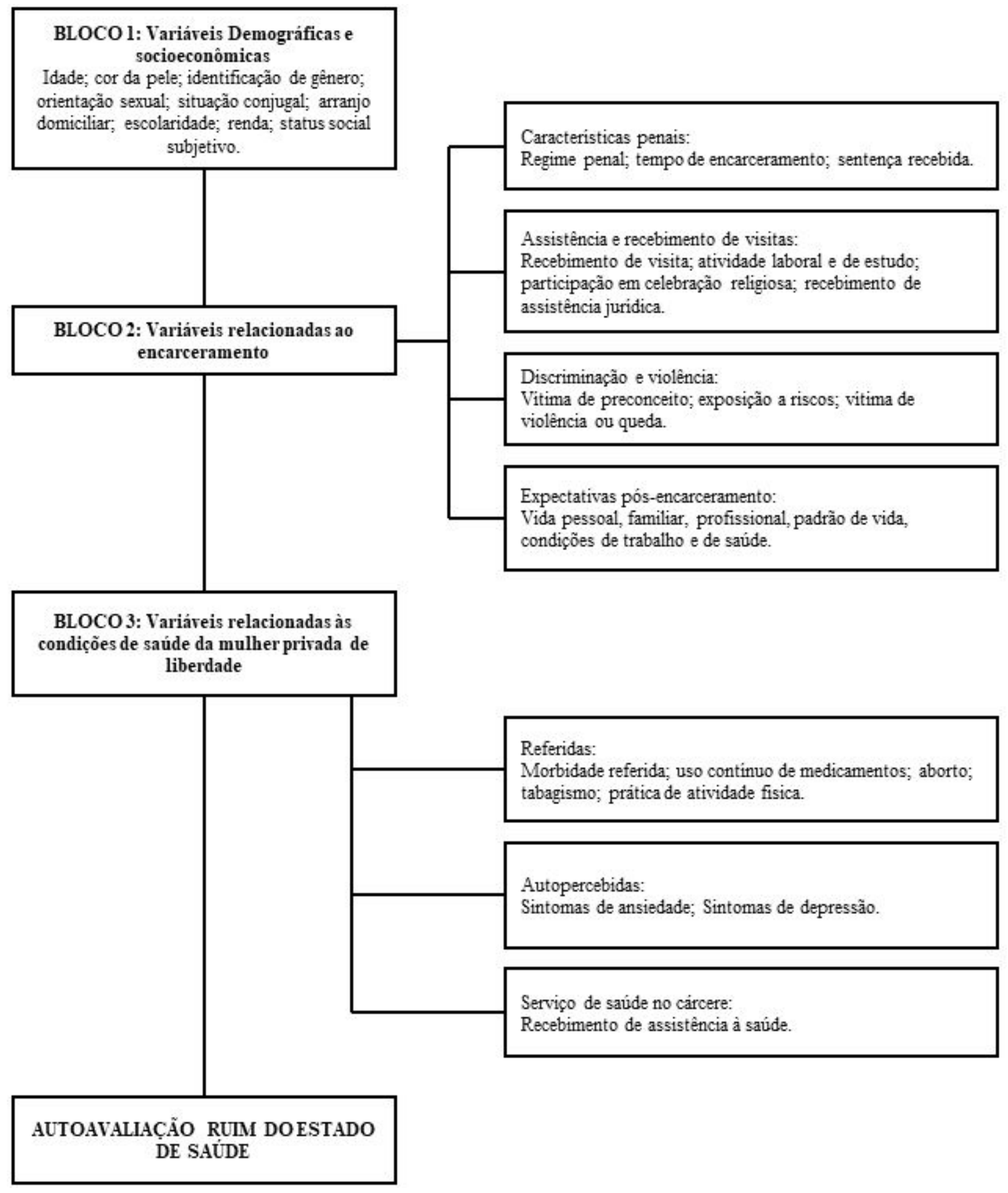

Figura 1. Modelo teórico de investigação dos efeitos das variáveis independentes sobre a autoavaliação ruim do estado de saúde por blocos hierarquizados.

Fonte: Os autores. 
Tabela 1. Características relacionadas ao encarceramento. Juiz de Fora, Minas Gerais, 2020 (n=99).

\begin{tabular}{|c|c|c|c|c|}
\hline \multirow{2}{*}{ Variável } & \multicolumn{2}{|c|}{ Autoavaliação ruim da saúde } & \multicolumn{2}{|c|}{ Autoavaliação boa da saúde } \\
\hline & $\mathbf{N}$ & $\%$ & $n$ & $\%$ \\
\hline \multicolumn{5}{|c|}{ Assistência e recebimento de visitas } \\
\hline \multicolumn{5}{|l|}{ Visita social } \\
\hline Sim & 16 & 51,6 & 44 & 64,7 \\
\hline Não & 15 & 48,4 & 24 & 35,3 \\
\hline \multicolumn{5}{|l|}{ Visita agendada } \\
\hline Sim & 7 & 22,6 & 18 & 26,5 \\
\hline Não & 24 & 77,4 & 50 & 73,5 \\
\hline \multicolumn{5}{|l|}{ Atividade laboral } \\
\hline Sim & 10 & 32,3 & 30 & 44,1 \\
\hline Não & 21 & 67,7 & 38 & 55,9 \\
\hline \multicolumn{5}{|l|}{ Estudo } \\
\hline Sim & 3 & 9,7 & 9 & 13,2 \\
\hline Não & 28 & 90,3 & 59 & 86,8 \\
\hline \multicolumn{5}{|l|}{ Assistência religiosa } \\
\hline Sim & 22 & 71,0 & 53 & 77,9 \\
\hline Não & 9 & 29,0 & 15 & 22,1 \\
\hline \multicolumn{5}{|l|}{ Assistência jurídica } \\
\hline Sim & 23 & 74,2 & 55 & 80,9 \\
\hline Não & 8 & 25,8 & 13 & 19,1 \\
\hline \multicolumn{5}{|c|}{ Tratamento discriminatório por outras detentas } \\
\hline \multicolumn{5}{|c|}{ Devido à condição de detenta } \\
\hline Sim & 12 & 38,7 & 19 & 27,9 \\
\hline Não & 19 & 61,3 & 49 & 72,1 \\
\hline \multicolumn{5}{|l|}{ Devido à cor da pele } \\
\hline Sim & 2 & 6,5 & 3 & 4,4 \\
\hline Não & 29 & 93,5 & 65 & 95,6 \\
\hline \multicolumn{5}{|l|}{ Devido à condição social } \\
\hline $\operatorname{Sim}$ & 12 & 38,7 & 14 & 20,6 \\
\hline Não & 19 & 61,3 & 54 & 79,4 \\
\hline \multicolumn{5}{|l|}{ Devido à orientação sexual } \\
\hline Sim & 7 & 22,6 & 9 & 13,2 \\
\hline Não & 24 & 77,4 & 59 & 86,8 \\
\hline \multicolumn{5}{|c|}{ Devido ao tipo de crime cometido } \\
\hline Sim & 7 & 22,6 & 11 & 16,2 \\
\hline Não & 24 & 77,4 & 57 & 83,8 \\
\hline \multicolumn{5}{|l|}{ Devido à aparência física } \\
\hline Sim & 12 & 38,7 & 12 & 17,6 \\
\hline Não & 19 & 61,3 & 56 & 82,4 \\
\hline \multicolumn{5}{|c|}{ Exposição a riscos e vítima de violência } \\
\hline \multicolumn{5}{|c|}{ Risco de agressão física } \\
\hline Sim & 18 & 58,1 & 31 & 45,6 \\
\hline Não & 13 & 41,9 & 37 & 54,4 \\
\hline
\end{tabular}

Fonte: Os autores. 
Tabela 1. Continued...

\begin{tabular}{|c|c|c|c|c|}
\hline \multirow{2}{*}{ Variável } & \multicolumn{2}{|c|}{ Autoavaliação ruim da saúde } & \multicolumn{2}{|c|}{ Autoavaliação boa da saúde } \\
\hline & $\mathbf{N}$ & $\%$ & $\mathbf{n}$ & $\%$ \\
\hline \multicolumn{5}{|l|}{ Risco de violência sexual } \\
\hline Não & 31 & 100,0 & 67 & 98,5 \\
\hline \multicolumn{5}{|l|}{ Risco de violência psicológica } \\
\hline \multicolumn{5}{|c|}{ Risco de ferimento por arma branca } \\
\hline Sim & 8 & 25,8 & 17 & 25,0 \\
\hline Não & 23 & 74,2 & 51 & 75,0 \\
\hline \multicolumn{5}{|c|}{ Risco de ferimento por arma de fogo } \\
\hline Sim & 9 & 29,0 & 13 & 19,1 \\
\hline Não & 22 & 71,0 & 55 & 80,9 \\
\hline \multicolumn{5}{|l|}{ Risco de explosão } \\
\hline Sim & 16 & 51,6 & 17 & 25,0 \\
\hline Não & 15 & 48,4 & 51 & 75,0 \\
\hline \multicolumn{5}{|l|}{ Vítima de agressão física } \\
\hline Sim & 8 & 25,8 & 8 & 11,8 \\
\hline Não & 23 & 74,2 & 60 & 88,2 \\
\hline \multicolumn{5}{|l|}{ Vítima de agressão verbal } \\
\hline Sim & 10 & 32,3 & 5 & 7,4 \\
\hline Não & 21 & 67,7 & 63 & 92,6 \\
\hline \multicolumn{5}{|l|}{ Tentativa de suicídio } \\
\hline Sim & 2 & 6,5 & 5 & 7,4 \\
\hline Não & 29 & 93,5 & 63 & 92,6 \\
\hline \multicolumn{5}{|l|}{ Tentativa de homicídio } \\
\hline Sim & 1 & 3,2 & 0 & 0,0 \\
\hline Não & 30 & 96,8 & 68 & 100,0 \\
\hline \multicolumn{5}{|c|}{ Expectativas pós-encarceramento } \\
\hline \multicolumn{5}{|c|}{ Vida pessoal } \\
\hline Boa & 22 & 71,0 & 49 & 72,1 \\
\hline Regular/ruim & 9 & 29,0 & 19 & 27,9 \\
\hline \multicolumn{5}{|l|}{ Vida familiar } \\
\hline Boa & 25 & 80,6 & 58 & 85,3 \\
\hline Regular/ruim & 6 & 19,4 & 10 & 14,7 \\
\hline Vida profissional & & & & \\
\hline
\end{tabular}

Fonte: Os autores. 
Tabela 1. Continuação...

\begin{tabular}{|c|c|c|c|c|}
\hline \multirow{2}{*}{ Variável } & \multicolumn{2}{|c|}{ Autoavaliação ruim da saúde } & \multicolumn{2}{|c|}{ Autoavaliação boa da saúde } \\
\hline & $\mathbf{N}$ & $\%$ & $n$ & $\%$ \\
\hline Boa & 17 & 54,8 & 44 & 64,7 \\
\hline Regular/ruim & 14 & 45,2 & 24 & 35,3 \\
\hline \multicolumn{5}{|l|}{ Padrão de vida } \\
\hline Boa & 11 & 35,5 & 37 & 54,4 \\
\hline Regular/ruim & 20 & 64,5 & 31 & 45,6 \\
\hline \multicolumn{5}{|l|}{ Condição de trabalho } \\
\hline Boa & 17 & 54,8 & 41 & 60,3 \\
\hline Regular/ruim & 14 & 45,2 & 27 & 39,7 \\
\hline \multicolumn{5}{|l|}{ Condição de saúde } \\
\hline Boa & 16 & 51,6 & 59 & 86,8 \\
\hline Regular/ruim & 15 & 48,4 & 9 & 13,2 \\
\hline
\end{tabular}

Fonte: Os autores.

Tabela 2. Características da população de estudo conforme variáveis relacionadas às condições de saúde. Juiz de Fora, Minas Gerais, 2020 ( $n=99)$.

\begin{tabular}{|c|c|c|c|c|}
\hline \multirow{2}{*}{ Variável } & \multicolumn{2}{|c|}{ Autoavaliação ruim da saúde } & \multicolumn{2}{|c|}{ Autoavaliação boa da saúde } \\
\hline & $n$ & $\%$ & $n$ & $\%$ \\
\hline \multicolumn{5}{|l|}{ Morbidade referida } \\
\hline Sim & 24 & 77,4 & 28 & 41,2 \\
\hline Não & 7 & 22,6 & 40 & 58,8 \\
\hline \multicolumn{5}{|c|}{ Uso contínuo de medicamentos } \\
\hline 1 a 3 medicamentos & 12 & 38,7 & 28 & 41,2 \\
\hline 4 ou mais medicamentos & 12 & 38,7 & 18 & 26,5 \\
\hline Nenhum & 7 & 22,6 & 22 & 32,4 \\
\hline \multicolumn{5}{|l|}{ Aborto } \\
\hline Espontâneo & 3 & 9,7 & 13 & 19,1 \\
\hline Provocado & 6 & 19,4 & 6 & 8,8 \\
\hline Nenhum & 22 & 71,0 & 49 & 72,1 \\
\hline \multicolumn{5}{|l|}{ Tabagismo } \\
\hline Sim & 25 & 80,6 & 47 & 69,1 \\
\hline Não & 6 & 19,4 & 21 & 30,9 \\
\hline \multicolumn{5}{|l|}{ Atividade física } \\
\hline Sim & 7 & 22,6 & 13 & 19,1 \\
\hline Não & 24 & 77,4 & 55 & 80,8 \\
\hline \multicolumn{5}{|l|}{ Sintomas de ansiedade } \\
\hline Presente & 30 & 96,8 & 45 & 66,2 \\
\hline Ausente & 1 & 3,2 & 23 & 33,8 \\
\hline \multicolumn{5}{|l|}{ Sintomas de depressão } \\
\hline Presente & 27 & 87,1 & 38 & 55,9 \\
\hline Ausente & 4 & 12,9 & 30 & 44,1 \\
\hline \multicolumn{5}{|l|}{ Assistência à saúde } \\
\hline Sim & 29 & 93,5 & 61 & 89,7 \\
\hline Não & 2 & 6,5 & 7 & 10,3 \\
\hline
\end{tabular}

Fonte: Os autores. 
de ansiedade $(p<0,002)$ e depressão $(p<0,005)$ (Tabela 3$)$. Nenhuma variável do Bloco 1 - referente às características sociodemográficas - apresentou significância estatística nesta análise.

No modelo de regressão múltipla, três variáveis permaneceram associadas à autoavaliação ruim do estado de saúde (Tabela 4). Das variáveis relacionadas ao encarceramento (Bloco 2), configurou-se como fator de risco a pior perspectiva em relação às condições de saúde após o cumprimento da pena (RP ajustada $=4,82 ;$ IC95\% 1,50-15,47). Entre as variáveis do nível mais proximal (Bloco 3 ), configuraram-se como fatores de risco a presença de sintomas de ansiedade (RP ajustada $=15,20$; IC95\% 1,79-128,92) e morbidade referida (RP ajustada $=4,11$; IC95\% 1,33-12,65).

Tabela 3. Razões de prevalência brutas e ajustadas entre os blocos hierárquicos para a ocorrência de autoavaliação ruim do estado de saúde em mulheres encarceradas. Juiz de Fora, Minas Gerais, 2020 (n=99).

\begin{tabular}{|c|c|c|c|c|c|}
\hline Variável & $\%$ & RP bruta (IC95\%) & $\mathbf{p}$ & RP ajustada (IC95\%) & $\mathbf{P}$ \\
\hline \multicolumn{6}{|c|}{ 2.3 - Variáveis relacionadas ao encarceramento: discriminação, violência ou queda } \\
\hline \multicolumn{3}{|c|}{$\begin{array}{l}\text { Tratamento inferior por outras } \\
\text { detentas devido à aparência física }\end{array}$} & \multirow{3}{*}{0,044} & & \multirow{3}{*}{0,324} \\
\hline Não & 25,3 & 1 & & 1 & \\
\hline Sim & 50 & $2,95(1,14-7,66)$ & & $1,75(0,58-5,34)$ & \\
\hline \multicolumn{5}{|l|}{ Risco de explosão } & \multirow{3}{*}{0,282} \\
\hline Não & 22,7 & 1 & \multirow[t]{2}{*}{0,018} & 1 & \\
\hline Sim & 48,5 & $3,20(1,31-7,82)$ & & $1,79(0,62-5,09)$ & \\
\hline \multicolumn{3}{|l|}{ Vítima de queda } & \multirow{3}{*}{0,004} & & \multirow{3}{*}{0,021} \\
\hline Não & 25 & 1 & & 1 & \\
\hline Sim & 66,7 & $6,00(1,84-19,56)$ & & $4,78(1,27-18,03)$ & \\
\hline \multicolumn{6}{|c|}{ 2.4 - Variáveis relacionadas ao encarceramento: expectativa pós-encarceramento } \\
\hline \multicolumn{3}{|c|}{ Condição de saúde } & \multirow{3}{*}{$<0,001$} & & \multirow{3}{*}{0,003} \\
\hline Boa & 21,3 & 1 & & 1 & \\
\hline Regular/ruim & 62,5 & $6,15(2,27-16,61)$ & & $5,29(1,78-15,66)$ & \\
\hline \multicolumn{6}{|c|}{ 3.1 - Variáveis relacionadas à saúde da mulher privada de liberdade: referidas } \\
\hline \multicolumn{3}{|l|}{ Morbidade referida } & \multirow{3}{*}{0,002} & & \multirow{3}{*}{0,001} \\
\hline Não & 14,9 & 1 & & 1 & \\
\hline Sim & 46,2 & $4,90(1,86-12,93)$ & & $6,22(2,18-17,75)$ & \\
\hline \multicolumn{6}{|c|}{3.2 - Variáveis relacionadas à saúde da mulher privada de liberdade: autopercebidas } \\
\hline \multicolumn{3}{|c|}{ Sintomas de ansiedade } & \multirow{3}{*}{0,002} & & \multirow{3}{*}{0,032} \\
\hline Ausente & 4,2 & 1 & & 1 & \\
\hline Presente & 40 & $15,33(1,97-119,67)$ & & $11,05(1,23-99,26)$ & \\
\hline \multicolumn{3}{|l|}{ Sintomas de depressão } & \multirow{3}{*}{0,005} & & \multirow{3}{*}{0,101} \\
\hline Ausente & 11,8 & 1 & & 1 & \\
\hline Presente & 41,5 & $5,33(1,68-16,90)$ & & $3,04(0,80-11,51)$ & \\
\hline
\end{tabular}

Fonte: Os autores.

Tabela 4. Análise de regressão múltipla em blocos hierarquizados para a ocorrência de autoavaliação ruim do estado de saúde em mulheres encarceradas. Juiz de Fora, Minas Gerais, 2020 ( $n=99)$.

\begin{tabular}{|c|c|c|c|c|c|}
\hline Variável & $\%$ & RP bruta (IC95\%) & $\mathbf{P}$ & RP ajustada (IC95\%) & $\mathbf{P}$ \\
\hline \multicolumn{6}{|c|}{2.3 - Variáveis relacionadas ao encarceramento: discriminação e violência } \\
\hline \multicolumn{6}{|l|}{ Vítima de queda } \\
\hline Não & 25 & 1 & \multirow[t]{2}{*}{0,004} & 1 & \multirow[t]{2}{*}{0,051} \\
\hline Sim & 66,7 & $6,00(1,84-19,56)$ & & $4,22(1,00-17,92)$ & \\
\hline \multicolumn{6}{|c|}{ 2.4 - Variáveis relacionadas ao encarceramento: expectativa pós-encarceramento } \\
\hline \multicolumn{3}{|c|}{ Condição de saúde } & \multirow{3}{*}{$<0,001$} & & \multirow{3}{*}{0,008} \\
\hline Boa & 21,3 & 1 & & 1 & \\
\hline Regular/Ruim & 62,5 & $6,15(2,27-16,61)$ & & $4,82(1,50-15,47)$ & \\
\hline
\end{tabular}

Fonte: Os autores. 
Tabela 4. Continuação...

\begin{tabular}{|c|c|c|c|c|c|}
\hline Variável & $\%$ & RP bruta (IC95\%) & $\mathbf{P}$ & RP ajustada (IC95\%) & $\mathbf{P}$ \\
\hline \multicolumn{6}{|c|}{ 3.1 - Variáveis relacionadas à saúde da mulher privada de liberdade: referidas } \\
\hline \multicolumn{6}{|c|}{ Morbidade referida } \\
\hline Não & 14,9 & 1 & \multirow[t]{2}{*}{0,002} & 1 & \multirow[t]{2}{*}{0,014} \\
\hline Sim & 46,2 & $4,90(1,86-12,93)$ & & $4,11(1,33-12,65)$ & \\
\hline \multicolumn{6}{|c|}{3.2 - Variáveis relacionadas à saúde da mulher privada de liberdade: autopercebidas } \\
\hline \multicolumn{5}{|c|}{ Sintomas de ansiedade } & \multirow{3}{*}{0,013} \\
\hline Ausente & 4,2 & 1 & 0,002 & 1 & \\
\hline Presente & 40 & $15,33(1,97-119,67)$ & & $15,20(1,79-128,92)$ & \\
\hline
\end{tabular}

Fonte: Os autores.

\section{DISCUSSÃO}

A prevalência de autoavaliação ruim da saúde na população estudada $(31,3 \%)$ foi significantemente inferior aos $61,5 \%$ encontrado entre mulheres acauteladas em uma unidade prisional do estado de São Paulo ${ }^{27}$ e superior à observada entre homens encarcerados na Noruega, em que $23 \%$ informaram estado de saúde ruim ou razoável ${ }^{22}$. Na comparação por sexo, estudos indicam que a pior autopercepção da saúde é mais frequente entre as mulheres do que entre os homens ${ }^{5,10,12,28}$. No entanto, no contexto das prisões, são escassos os estudos que objetivaram investigar a autopercepção da saúde e fatores associados entre privados de liberdade, o que limita a comparação neste grupo. É possível que características intrínsecas à organização das instituições penais, que são diferentes entre os locais dos estudos, o porte das cidades e as diferenças nas leis de execução penal, quando analisamos a diferença com outros países, também influenciem na autopercepção da saúde dos indivíduos sob custódia 20,21 .

Na população geral feminina, há pesquisas que identificam prevalências de autoavaliação ruim da saúde em torno de $40 \%{ }^{9,17}$. No entanto, utilizaram categorias para o desfecho diferentes da empregada neste trabalho. Dentre aqueles que utilizaram categorias semelhantes, destacam-se importantes estudos de base populacional. Estudo realizado a partir de dados da Pesquisa Nacional de Saúde (PNS) de 2013, inquérito domiciliar de base populacional, realizado pelo Instituto Brasileiro de Geografia e Estatística (IBGE), em parceria com o Ministério da Saúde, apontou que 37,4\% das mulheres autoavaliaram a saúde como regular, ruim ou muito ruim ${ }^{10}$. Na Pesquisa Mundial de Saúde, esse percentual foi de $52,5 \%{ }^{7}$ e na PNS de $37,6 \%{ }^{12}$. Considerando a soma das categorias supracitadas para o desfecho autoavaliação ruim da saúde, a prevalência encontrada no presente estudo foi, de forma geral, menor do que a identificada na população geral. No entanto, o percentual de acauteladas que referiu saúde ruim ou muito ruim foi maior do que o encontrado em alguns estudos ${ }^{10,12,29}$, evidenciando que a forma de categorização do desfecho influencia em sua interpretação final, sendo que mulheres em situação de cárcere podem apresentar pior autoavaliação da saúde do que quando comparadas às mulheres da população geral.

A falta de padronização na utilização e análise do indicador prejudica a comparação entre os estudos. As diferenças entre os achados estão, em parte, relacionadas às diferentes denominações das categorias de respostas à pergunta de autoavaliação da saúde, aos distintos agrupamentos das categorias de reposta, às características da população estudada e à localização da pergunta nos instrumentos utilizados ${ }^{13,29}$.

Em relação às variáveis sociodemográficas, idade, status social subjetivo, nível de escolaridade e de renda, diferentemente dos resultados de estudos nacionais ${ }^{12}$ e internacionais ${ }^{5,16-19}$, este estudo não encontrou associações com a autoavaliação ruim da saúde. É possível que isso tenha ocorrido, devido à homogeneidade da amostra para esse grupo de variáveis, bem como ao tamanho reduzido da mesma.

Dentre as variáveis relacionadas ao encarceramento, a pior percepção do estado de saúde foi mais frequente entre aquelas que declararam ter sofrido tratamento inferior por outras detentas, devido à aparência física entre as que acreditam no risco de explosão da unidade prisional, as que foram vítimas de queda durante o cumprimento da pena e entre aquelas que relataram pior perspectiva em relação à sua condição de saúde após o período do encarceramento. Esses achados atestam que a autopercepção da saúde está, dentre outros aspectos, sob influência do ambiente físico e social, corroborando outros estudos ${ }^{5,11,15,30}$, evidenciando a influência do encarceramento na percepção de saúde das acauteladas.

Pesquisa realizada nas prisões do estado do Rio de Janeiro mostrou que as mulheres relataram sofrer mais discriminação por parte de outras detentas do que os homens por outros encarcerados $^{22}$. Também foi observada maior proporção de quedas entre as mulheres presas, sendo esse percentual de $21,7 \%$, enquanto que, entre os homens, foi de $12,9 \%{ }^{22}$. Não há na literatura estudos que analisem a associação entre as variáveis supracitadas e a autopercepção de saúde no âmbito das prisões. No entanto, a maior ocorrência de quedas entre mulheres e sua associação com a pior percepção de saúde já foi descrita na população idosa ${ }^{31}$. 
Algumas particularidades encontradas no local onde o estudo foi conduzido podem ter contribuído para a maior ocorrência de algumas observações. As ocorrências de queda podem estar relacionadas ao fato de os alojamentos disporem de "triliches", como também em decorrência de problemas de convívio com outras detentas, uma vez que as mesmas são alocadas em alojamentos com capacidade para até 30 acauteladas. Já a preocupação quanto ao risco de explosão da unidade prisional pode estar associada às recorrentes quedas de energia, conforme relatado por algumas participantes. $O$ fato é que a associação desses eventos com a autoavaliação do estado de saúde enfatiza a necessidade de se compreender a saúde dessa população sob a ótica de diferentes determinantes.

Nesse sentido, diversos estudos apontam que o encarceramento tem influência direta e indireta nos problemas de saúde física e mental dos indivíduos privados de liberdade ${ }^{2,3,22,32}$. Sendo assim, é aceitável que os efeitos deletérios do cárcere influenciem na perspectiva de vida após o período de acautelamento, principalmente no que tange às condições de saúde futuras. Os resultados deste estudo revelaram associação entre pior perspectiva em relação às condições de saúde futuras e a autoavaliação ruim da saúde, no entanto tal relação ainda não foi descrita na literatura.

O relato de morbidades se associou à pior percepção de saúde, ratificando apontamentos da literatura, 5,9,12,18,29. Shooshtari et al. ${ }^{18}$ confirmam que autoavaliação de saúde é dinâmica e multidimensional, contudo, reforça a importância da dimensão física, o que inclui as questões de bem-estar psicológico e saúde mental no processo de autoavaliação do estado de saúde.

Um elevado percentual de mulheres referiu sintomas de ansiedade e depressão, correspondendo a, respectivamente, $75,8 \%$ e $65,7 \%$ da população estudada. Altas prevalências de transtornos mentais na população carcerária e, especialmente, entre as mulheres, já foram encontradas por outros autores ${ }^{3,33,34}$. Pesquisas anteriores encontraram associações entre a saúde mental e o bem-estar psicológico e a autoavaliação da saúde ${ }^{5,35,36}$, e nossos resultados confirmam a importância do sofrimento psíquico em relação a este desfecho.

Os resultados encontrados indicam que, além da dimensão biológica da doença, o contexto em que o indivíduo está inserido é um fator preponderante para o processo de adoecimento. Além disso, há de se considerar que as iniquidades em saúde observadas nessa população, sobretudo na perspectiva de gênero, são decorrentes, não somente das condições impostas pelo encarceramento, mas também devido à vulnerabilidade prévia que o grupo apresenta e que tende a se exacerbar com o encarceramento. Assim, nota-se uma cascata de eventos que culminam, não só na intensificação das desigualdades sociais, mas também em piores condições de saúde. Esse contexto nos convoca a refletir sobre a importância do debate em torno do modelo de determinação social do processo saúde adoecimento e a compreensão de que os níveis de organização da sociedade expressam os níveis de saúde da população.
As taxas de prevalência de tuberculose nessa população, muito superiores às encontradas na população geral, retratam as iniquidades em saúde presentes em nossa sociedade. As condições precárias dos presídios brasileiros, marcadas pela superlotação, ventilação deficiente, acesso limitado ao serviço de saúde, desnutrição, uso de álcool e outras drogas ${ }^{37}$, favorece a disseminação dos patógenos e o comprometimento da saúde das pessoas privadas de liberdade. As iniquidades em saúde que envolvem a população carcerária justificam que a necessidade de atenção e cuidado não são iguais para todos os segmentos da população, confirmando a importância de se garantir mais direitos aos que têm maiores necessidades ${ }^{38}$.

Para a autora, o tema da equidade em saúde é indissociável das questões de justiça social, o que implica, inclusive, um olhar diferenciado sobre a população carcerária feminina, haja vista a vulnerabilidade prévia do grupo, apoiada na convergência dos marcadores de raça, gênero e classe para aplicação da punibilidade, e o fato de que as unidades prisionais destinadas ao público feminino são, em sua maior parte, adequações de estabelecimentos originalmente masculinos, cuja infraestrutura não é apropriada para atender às especificidades da mulher ${ }^{39}$. Não obstante, as mulheres encarceradas são, de fato, mais acometidas por agravos à saúde do que as mulheres da população geral ${ }^{40}$.

Meireles e colaboradores $(2015)^{11}$ reforçam a necessidade de inclusão de variáveis do ambiente físico e social dos indivíduos para análise da autoavaliação da saúde. Assim, o uso do indicador no contexto carcerário parece ser um componente de análise importante, mas que requer, para fins de comparação, padronização. Sugerimos a realização de outros estudos epidemiológicos que contemplem uma amostra representativa desta população e que aprofundem as análises aqui propostas, haja vista que se constituem em um importante elemento de investigação para os desfechos de saúde.

Diante do crescimento exponencial da população carcerária feminina, emerge a necessidade de compreensão da dinâmica de saúde desse grupo, cujo perfil confirma o processo de vulnerabilização pelo qual vem sendo expostas ao longo dos anos e que tendem a se intensificar com o encarceramento.

\section{CONSIDERAÇÕES FINAIS E IMPLICAÇÕES PARA A PRÁTICA}

A autoavaliação ruim da saúde foi relatada por aproximadamente um terço das mulheres e está associada a variáveis relacionadas às condições de saúde - morbidade referida e sintomas de ansiedade, e do encarceramento - pior perspectiva em relação às condições de saúde pós-encarceramento. Esses resultados confirmam que a autopercepção da saúde está sob influência de diferentes aspectos, reforçando que a saúde das mulheres encarceradas deve ser compreendida sob a ótica do conceito ampliado de saúde.

Os resultados deste estudo devem ser interpretados dentro do contexto de suas limitações. Entre elas está o desenho 
metodológico, que não permite que se façam inferências de causa e efeito sobre as associações encontradas. A população estudada apresenta peculiaridades, como receio de retaliações por funcionários e/ou outras detentas, insegurança emocional e medo de interferências negativas no cumprimento da pena, que podem interferir em resultados mais fidedignos. Assim, é necessário considerar a possibilidade de respostas que não correspondem à realidade, mesmo diante do rigor metodológico adotado. Além disso, a coleta de dados foi interrompida em decorrência da pandemia de COVID-19, reduzindo o tamanho da amostra. Cabe ressaltar ainda que o local de condução do estudo foi inaugurado a menos de dois anos e conta com uma melhor infraestrutura e condições assistenciais, quando comparado ao local onde as acauteladas cumpriam a pena anteriormente, o que pode ter influenciado positivamente as respostas sobre alguns dados pesquisados.

Ainda assim, destaca-se a importância deste trabalho, cujos resultados apresentados trazem, sob a ótica do conceito ampliado de saúde, informações relevantes sobre a saúde das mulheres em privação de liberdade, possibilitando também a identificação dos fatores associados à autoavaliação ruim da saúde nessa parcela negligenciada da população. A atenção à saúde da mulher privada de liberdade se apresenta como um grande desafio para a saúde pública na atualidade, tornando necessária a compreensão das questões que permeiam a realidade do grupo.

Este estudo tem potencial para contribuir na minimização dos impactos sociais e à saúde física e mental que afligem o público em questão, subsidiando estratégias para o enfrentamento às iniquidades em saúde.

\section{CONTRIBUIÇÕES DOS AUTORES}

Desenho do estudo. Lidiane Castro Duarte de Aquino. Isabel Cristina Gonçalves Leite. Danielle Teles da Cruz.

Coleta de dados. Lidiane Castro Duarte de Aquino. Danielle Teles da Cruz.

Análise de dados. Lidiane Castro Duarte de Aquino. Bruna Gomes de Souza. Cosme Rezende Laurindo. Isabel Cristina Gonçalves Leite. Danielle Teles da Cruz.

Interpretação dos resultados. Lidiane Castro Duarte de Aquino. Bruna Gomes de Souza. Cosme Rezende Laurindo. Isabel Cristina Gonçalves Leite. Danielle Teles da Cruz.

Redação e revisão crítica do manuscrito. Lidiane Castro Duarte de Aquino. Bruna Gomes de Souza. Cosme Rezende Laurindo. Isabel Cristina Gonçalves Leite. Danielle Teles da Cruz.

Aprovação da versão final do artigo. Lidiane Castro Duarte de Aquino. Bruna Gomes de Souza. Cosme Rezende Laurindo. Isabel Cristina Gonçalves Leite. Danielle Teles da Cruz.

Responsabilidade por todos os aspectos do conteúdo e a integridade do artigo publicado. Lidiane Castro Duarte de Aquino. Bruna Gomes de Souza. Cosme Rezende Laurindo. Isabel Cristina Gonçalves Leite. Danielle Teles da Cruz.

\section{EDITOR ASSOCIADO}

Cristina Rosa Baixinho (1)

\section{EDITOR CIENTÍFICO}

\author{
Ivone Evangelista Cabral (D)
}

\section{REFERÊNCIAS}

1. Santos $\mathrm{T}$, organizador. Levantamento nacional de informações penitenciárias - INFOPEN mulheres. 2. ed. Brasília: Departamento Penitenciário Nacional, Ministério da Justiça e Segurança Pública 2017.

2. Audi CAF, Santiago SM, Andrade MGG, Francisco PMS. Inquérito sobre condições de saúde de mulheres encarceradas. Saúde Debate. 2016;40(109):112-24. http://dx.doi.org/10.1590/0103-1104201610909.

3. Baranyi G, Scholl C, Fazel S, Patel V, Priebe S, Mundt AP. Severe mental illness and substance use disorders in prisoners in low-income and middle-income countries: a systematic review and meta-analysis of prevalence studies. Lancet Glob Health. 2019;7(4):e461-71. http:// dx.doi.org/10.1016/S2214-109X(18)30539-4. PMid:30879509.

4. Leite ASQ, Lira Ferreira C, Silva KLL, Lima ADP, Leão LD, Carvalho YNFS. Questões de gênero nas instituições prisionais: um olhar a partir do projeto grupo de diálogo universidade-cárcere-comunidade. Pretextos [Internet]. 2020; [citado 2021 jul 15];5(9):598-617. Disponível em: http:// periodicos.pucminas.br/index.php/pretextos/article/view/22469

5. Ross J, Field C, Kaye S, Bowman J. Prevalence and correlates of low self-reported physical health status among prisoners in New South Wales, Australia. Int J Prison Health. 2019;15(2):192-206. http://dx.doi. org/10.1108/IJPH-06-2018-0039. PMid:31172857.

6. DeSalvo KB, Bloser N, Reynolds K, He J, Muntner P. Mortality prediction with a single general selfrated health question: a meta-analysis. J Gen Intern Med. 2006;21(3):267-75. http://dx.doi.org/10.1111/j.15251497.2005.00291.x. PMid:16336622.

7. Szwarcwald CL, Souza-Júnior PRB, Esteves MAP, Damacena GN Viacava F. Socio-demographic determinants of self-rated health in Brazil. Cad Saude Publica. 2005;21(1, Supl.):54-64. http://dx.doi.org/10.1590/ S0102-311X2005000700007. PMid:16462997.

8. Idler EL, BenyaminiY. Self-rated health and mortality: a review of twentyseven community studies. J Health Soc Behav. 1997;38(1):21-37. http:// dx.doi.org/10.2307/2955359. PMid:9097506.

9. Pavão ALB, Werneck GL, Campos MR. Autoavaliação do estado de saúde e a associação com fatores sociodemográficos, hábitos de vida e morbidade na população: um inquérito nacional. Cad Saude Publica. 2013:29(4):723-34. http://dx.doi.org/10.1590/S0102-311X2013000800010. PMid:23568302.

10. Sousa JL, Alencar GP, Antunes JLF, Silva ZP. Marcadores de desigualdade na autoavaliação da saúde de adultos no Brasil, segundo o sexo. Cad Saude Publica. 2020;36(5):e00230318. http://dx.doi.org/10.1590/0102311x00230318. PMid:32490914.

11. Meireles AL, Xavier CC, Andrade ACS, Friche AAL, Proietti FA, Caiaffa WT. Autoavaliação da saúde em adultos urbanos, percepção do ambiente físico e social e relato de comorbidades: estudo saúde em Beagá. Cad Saude Publica. 2015;31(1):120-35. http://dx.doi.org/10.1590/0102311X00076114. PMid:26648368.

12. Szwarcwald CL, Damacena GN, Borges PR Jr, Almeida WS, Lima LTM, Malta DC et al. Determinantes da autoavaliação de saúde no Brasil e a influência dos comportamentos saudáveis: resultados da Pesquisa Nacional de Saúde. 2013. Rev Bras Epidemiol. 2015;18(2, Supl. 2):33-44. http://dx.doi.org/10.1590/1980-5497201500060004 PMid:27008601.

13. Belmonte JMMM, Pivetta NRS, Batistoni SST, Neri AL, Borim FSA. The association between self-rated health and functional capacity indicators. Geriatr. Gerontol. Aging. 2017;11(2):61-7. http://dx.doi.org/10.5327/ Z2447-211520171700021.

14. Szwarcwald CL, Damacena GN, Barros MBA, Malta DC, Souza PRB Jr Azevedo LO et al. Factors affecting Brazilians' self-rated health during 
the COVID-19 pandemic. Cad Saude Publica. 2021;37(3):e00182720. http://dx.doi.org/10.1590/0102-311x00182720. PMid:33950075.

15. Jylhä $M$. What is self-rated health and why does it predict mortality? Towards a unified conceptual model. Soc Sci Med. 2009;69(3):307-16. http://dx.doi.org/10.1016/j.socscimed.2009.05.013. PMid:19520474.

16. Friestad C. Socio-economic status and health in a marginalized group: the role of subjective social status among prison inmates. Eur J Public Health. 2010;20(6):653-8. http://dx.doi.org/10.1093/eurpub/ckp242. PMid:20123684.

17. Silva VH, Rocha JSB, Caldeira AP. Fatores associados à autopercepção negativa de saúde em mulheres climatéricas. Cien Saude Colet. 2018;23(5):1611-20. http://dx.doi.org/10.1590/1413-81232018235.17112016. PMid:29768614.

18. Shooshtari S, Menec V, Tate R. Comparing Predictors of Positive and Negative Self-Rated Health Between Younger (25-54) and Older (55+) Canadian Adults: A Longitudinal Study of Well-Being. Res Aging. 2007;29(6):512-54. http://dx.doi.org/10.1177/0164027507305729.

19. Operario D, Adler NE, Williams DR. Subjective Social Status: reliability and predictive utility for global health. Psychol Health. 2004;19(2):23746. http://dx.doi.org/10.1080/08870440310001638098.

20. Vogel CE, Barry LC. Self-rated health as a predictor os depression and axiety in older adult inmates. Innov Aging. 2019;3(Supl. 1):S166. http:// dx.doi.org/10.1093/geroni/igz038.593.

21. Yu SSV, Sung HE, Mellow J, Koenigsmann CJ. Self-Perceived Health Improvements Among Prison Inmates. J Correct Health Care. 2015;21(1):59-69. http://dx.doi.org/10.1177/1078345814558048. PMid:25559631.

22. Minayo MCS, Constantino P. Deserdados Sociais: condições de vida e saúde dos presos do estado do Rio de Janeiro. Rio de Janeiro: Ed. FioCruz; 2015.

23. Löwe B, Wahl I, Rose M, Spitzer C, Glaesmer H, Wingenfeld K et al. A 4-item measure of depression and anxiety: validation and standardization of the Patient Health Questionnaire-4 (PHQ-4) in the general population. J Affect Disord. 2010;122(1-2):86-95. http://dx.doi.org/10.1016/j. jad.2009.06.019. PMid:19616305.

24. Kroenke K, Spitzer RL, Williams JBW, Lowe B. An ultra-brief screening scale for anxiety and depression: the PHQ-4. Psychosomatics. 2009;50(6):613-21. http://dx.doi.org/10.1176/appi.psy.50.6.613. PMid:19996233.

25. Sanchón-Macias MV, Prieto-Salceda D, Bover-Bover A, Gastaldo D. Relação entre status social subjetivo e saúde percebida entre mulheres imigrantes latino-americanas. Rev Lat Am Enfermagem. 2013;21(6):13539. http://dx.doi.org/10.1590/0104-1169.2943.2374. PMid:24402346.

26. Victora CG, Huttly SR, Fuchs SC, Olinto MT. The role of conceptual frameworks in epidemiological analysis: a hierarchical approach. Int $J$ Epidemiol. 1997;26(1):224-7. http://dx.doi.org/10.1093/ije/26.1.224. PMid:9126524.

27. Bezerra C, Fernandes RAQ. Perfil social e de saúde de mulheres apenadas de uma penitenciária da cidade de São Paulo. Perspect Medicas. 2015;26(2):2130. http://dx.doi.org/10.6006/perspectmed.20150203.7233250448.
28. Franks P, Gold MR, Fiscella K. Sociodemographics, self-rated health, and mortality in the US. Soc Sci Med. 2003;56(12):2505-14. http:// dx.doi.org/10.1016/S0277-9536(02)00281-2. PMid:12742613.

29. Barros MBA, Zanchetta LM, Moura EC, Malta DC. Auto-avaliação da saúde e fatores associados, Brasil, 2006. Rev Saude Publica. 2009;43(Supl. 2):27-37. http://dx.doi.org/10.1590/S0034-89102009000900005 PMid:19936496.

30. Santos SM, Chor D, Werneck GL, Coutinho ESF. Associação entre fatores contextuais e auto-avaliação de saúde: uma revisão sistemática de estudos multinível. Cad Saude Publica. 2007;23(11):2533-54. http:// dx.doi.org/10.1590/S0102-311X2007001100002. PMid:17952247.

31. Siqueira FV, Facchini LA, Piccini RX, Tomasi E, Thumé E, Silveira DS et al. Prevalência de quedas em idosos e fatores associados. Rev Saude Publica. 2007;41(5):749-56. http://dx.doi.org/10.1590/S003489102007000500009. PMid:17923896.

32. Freudenberg N. Jails, prisons and the health of urban population: a review of the impact of the correctional system on community health. J Urban Health. 2001;78(2):214-35. http://dx.doi.org/10.1093/jurban/78.2.214. PMid:11419576.

33. Constantino P, Assis SG, Pinto LW. O impacto da prisão na saúde mental dos presos do estado do Rio de Janeiro, Brasil. Cien Saude Colet. 2016;21(7):2089-100. http://dx.doi.org/10.1590/141381232015217.01222016. PMid:27383343.

34. Fazel S, Seewald K. Severe mental illness in 33588 prisoners worldwide: systematic review and metaregression analysis. $\mathrm{Br} \mathrm{J}$ Psychiatry. 2012;200(5):364-73. http://dx.doi.org/10.1192/bjp.bp.111.096370. PMid:22550330.

35. BenyaminiY, Leventhal EA, Leventhal H. Gender differences in processing information in making self-assessments of health. Psychosom Med. 2000;62(3):354-64. http://dx.doi.org/10.1097/00006842-20000500000009. PMid: 10845349.

36. Idler EL, Hudson SV, Leventhal $H$. The meanings of self-ratings of health: a qualitative and quantitative approach. Res Aging. 1999;21(3):458-76 http://dx.doi.org/10.1177/0164027599213006.

37. Valenca MS, Possuelo LG, Cezar-Vaz MR, Silva PEA. Tuberculose em presídios brasileiros: uma revisão integrativa da literatura. Cien Saude Colet. 2016;21(7):2147-60. http://dx.doi.org/10.1590/141381232015217.16172015. PMid:27383348.

38. Magalhães R. Desigualdades sociais e equidade em saúde. In: Ministério da Saúde. Fundação Oswaldo Cruz, Núcleo de Estudos em Direitos Humanos e Saúde, organizador. Saúde e direitos humanos. Brasília: Ministério da Saúde; 2004. p. 61-8.

39. Silva MVM, organizador. Relatório temático sobre mulheres privadas de liberdade - junho de 2017. Brasília: Ministério da Justiça e Segurança Pública. Departamento Penitenciário Nacional; 2019.

40. Almeida PRC, Soares RSC, Coura AS, Cavalcanti AL, Dutra MOM, Lima TMA. Condição de saúde de mulheres privadas de liberdade: uma revisão integrativa. Rev Bras Ciênc Saúde. 2015;19(1):73-80. http://dx.doi.org/10.4034/RBCS.2015.19.01.12.

\footnotetext{
${ }^{a}$ Artigo extraído da dissertação de mestrado Autoavaliação do estado de saúde em mulheres privadas de liberdade em Juiz de Fora - MG, de autoria de Lidiane Castro Duarte de Aquino, com orientação de, Danielle Teles da Cruz. Universidade Federal de Juiz de Fora. Programa de Pós-Graduação em Saúde Coletiva. Ano de defesa: 2020
} 